\title{
Microextensometry and mechanical behaviour of Haversian cortical bone
}

\author{
Laurent Henry*,** — Thierry Hoc* — Elisa Budyn*** \\ * MSSMAT, Ecole Centrale Paris CNRS UMR 8579 \\ Grande Voie des Vignes \\ F-92295 Chatenay-Malabry, France \\ ** Institut curie \\ 11 rue Pierre et Marie Curie, F-75005, France \\ Laurent.henry@curie.fr \\ *** Department of Mechanical and Industrial Engineering \\ University of Illinois at Chicago, 842 West Taylor Street \\ Chicago IL 60607, USA
}

\begin{abstract}
It is well known that bone microarchitecture is mainly the result of the bone remodelling process. However, a mechanistic framework describing how the microstructure affects the mechanical behaviour of bone is still lacking. Therefore, tools to quantify bone quality at the microstructure scale are required. To address this problem, the present study focused on the measurements of the local strains over a large microstructure area using microextensometry. First, the relationship between the local strains and the mineral content was examined. Then, the local strains were used to feed an inverse approach performed by finite elements. The results show there is no correlation between the strain and the mineral content. Moreover, the implementation of the mineral content in finite elements simulations gave a better estimate of the experimental strain field.

RÉSUMÉ. Avec le vieillissement de la population, la prédiction du risque fracturaire et donc $d u$ comportement mécanique du tissu osseux, est devenue un enjeu majeur. S'il est maintenant évident que le remodelage osseux est à l'origine de sa microarchitecture, son influence sur le comportement mécanique est loin d'être élucidé. Il est nécessaire de se doter d'outils susceptibles de donner des informations sur la qualité mécanique du tissu à cette échelle. Pour répondre à cette problématique, une étude des déformations locales de la microstructure utilisant la microextensométrie est présentée. Les résultats sont analysés en termes de contenu minéral local mais aussi comme données d'entrées d'une approche inverse réalisée par éléments finis. Les résultats montrent qu'il n'y a pas de lien direct entre les concentrations de déformations et celles du contenu minéral. De plus, l'introduction de l'hétérogénéité de contenu minéral dans une simulation éléments finis permet de mieux reproduire les champs expérimentaux.
\end{abstract}

KEYWORDS: cortical bone, microextensometry, finite element, multiscale method. MOTS-CLÉS: tissu osseux cortical, microextensométrie, elements finis, méthode multiéchelle.

DOI:10.3166/EJCM.18.93-104 (C) 2009 Lavoisier, Paris

EJCM - 18/2009. Numerical models in biomechanics, pages 93 to 104 


\section{Introduction}

Human cortical bone is a composite material which mechanical properties are highly dependent on its hierarchical organization (Cowin, 2001; Katz, 1980). At the molecular scale, cortical bone is composed of an organic phase (collagen I fibrils) synthesized by the osteoblasts and an inorganic phase composed mainly of hydroxyapatite in the form of small crystallites. In human bones, the admixture of these two phases fibrils results in fibers that rearrange into a densely packed concentric lamellar structure (osteon) that is embedded into an interstitial matrix. Typically, an osteon is a cylinder measuring approximately 250-300 $\mathrm{m}$ in diameter and few millimeters in length with a central canal, the Haversian canal, for vascularization and innervation. Each osteon is composed of a set of concentric subunits (lamellae) while the interstitial system is made of fragments of old and partially remodeled osteons.

Moreover, bone is a living tissue that can adapt its structure and geometry to its mechanical environment (Currey, 2003). Bone remodeling is a multi-cellular phenomenon that permits this adaptation as well as the repair of micro-damages (Burr et al., 1997). Cellular mechanical environment is certainly one of the key parameters that triggers and regulates this process.

The mechanical properties of cortical bone have been studied extensively at the macrostructural level (Hobatho et al., 1991). In particular, the relationship existing between intracortical porosity or mineral density and macroscopic mechanical properties has been well documented on a large variety of specimens. At the microscale, e.g. the size of an osteon or less, two types of approaches have been performed. The first consisted in mechanical test on single osteons (Ascenzi et al., 1990). The second was based on nanoindentation, which gave access to the mechanical properties of bone at a scale less than one micrometer (Rho et al., 1999). However, many important properties of bone cannot be deduced solely through direct up scaling from the osteon behaviour. Damage, as well bone remodeling, cell activity... depend strongly on the local mechanical states that are beyond the scope of macroscopic approaches. In that case, the knowledge of the mechanical behaviour at the microstructure scale is always lacking.

Microextensometry provides a new tool for addressing this difficult problem. For the last two decades, the development of microextensometry techniques, combined with image correlation analysis, have made it possible to accurately measure displacements and strain fields on the surface of a sample. In particular the use of light microscopy now allows the characterization of the micro-strain of a large area (several square millimeters) in relation with the local microstructure. In parallel the Backscattered Electron Imaging (BSE) performed in a SEM-FEG gives access to a parameter directly related to the mineral content with a very high resolution. This technique makes possible to combine microextensometry measurement with the characterization of the degree of local heterogeneity and mineralization. With use of 
finite elements, meaningful comparisons can now be performed between accurate strain field measurements and their simulated counterparts.

The aim of our study is to quantify the mechanical properties of a cortical bone sample at the osteon scale. The different techniques, backscattered electron imaging and microextensometry, used in the present study are described in the first part. The second part is devoted to studying the heterogeneities of the local axial strain and the mineral content inside the microstructure. In the third part, the mechanical behaviour at osteon scale is determined through an inverse approach based on the local axial strain field accessed by microextensometry. The results are discussed in the last part.

\section{Materials and methods}

\subsection{Bone sample preparation}

First we analyzed the mechanical properties of mature bovine cortical. The objective of this experimental part study was to characterize the intrinsic local mechanical and the mineral properties within a well-defined bone. All bone samples were obtained from the posterior area of the mid femoral diaphysis of a single femur. Fresh specimens were cut with a diamond saw into prisms $(5 \mathrm{~mm}$ in length; $2 \times$ $2 \mathrm{~mm}$ in cross-section). All surfaces of these samples were ground with sandpaper (grit \#2400). The observed surfaces under optical microscopy were polished using 1$\mu \mathrm{m}$ diamond powder. Compression tests were performed on three samples in longitudinal and transversal direction. The results are reported only for one of each. All samples exhibited a Haversian microstructure. The Cartesian coordinate system chosen has the 1-axis (radial axis) parallel to the endosteal-periostal direction and the 3-axis (longitudinal axis) parallel to the osteonal axis. The 2-axis is in the circumferential direction of the cortical bone specimens tested.

\subsection{Backscattered electron (BSE) imaging}

The BSE imaging was performed using a SEM-FEG (LEO) equipped with a four quadrants BSE detector. The accelerating voltage was $20 \mathrm{kV}$, at a working distance of $15 \mathrm{~mm}$ and an approximately 100 times magnification. After mechanical testing, the bone samples were air dried, and the surfaces were carbon-coated using vacuum evaporation. However, the grey level distribution on BSE images depends on brightness (offset level control of the signal) and contrast (gain control of signal amplification). In order to avoid artefacts related to changes in the setting of the BSE imaging system, which may arise when taking multiple images, only one highresolution image was recorded for each sample $(3072 \times 2304$ pixels resolution, using 256 grey levels). 


\subsection{Microextensometry and mechanical tests}

Full field measurement techniques allow quantitative measurements of strain fields in heterogeneous materials. Digital image correlation (DIC) allows accurate measurements of the local displacements. This method compares two homologous domains, namely, correlation domains, one on the reference image (undeformed specimen) and the other on the deformed digital image by optimizing various correlation coefficients; in the present study, the correlation domain was fixed to $60 \times 60$ pixels. Details of the numerical background, which is beyond the scope of this paper, are described in reference (Doumalin et al., 2003).

For cortical bone, the local contrast required by the correlation technique and the quantification of the displacement field was provided by the intrinsic microstructure of this tissue. The displacement of microstructural features visible on the bone surface was determined from images obtained using a reflection optical microscope (OM) equipped with a high-resolution camera providing digital images with a 2000 $\times 1500$ pixels resolution $\left(1.3 \times 1 \mathrm{~mm}^{2}\right)$. Images of deformed bone samples were taken during testing under uniaxial compression with a custom-made compression device located on the focal plane of the optical microscope; this arrangement allowed direct observation of the surface of the bone specimen (see Figure 1). The compression tests were performed in the transversal direction 1 and the longitudinal direction, with an imposed strain rate of $10^{-4} \mathrm{~s}^{-1}$.

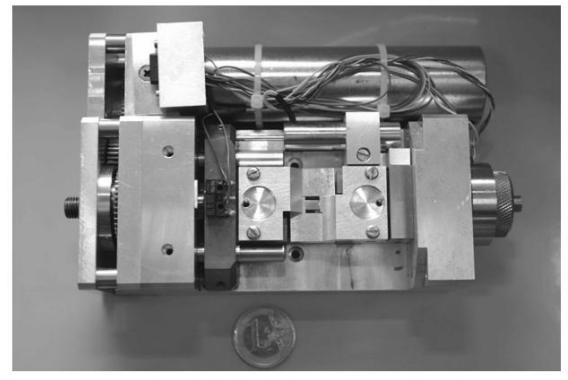

(a)

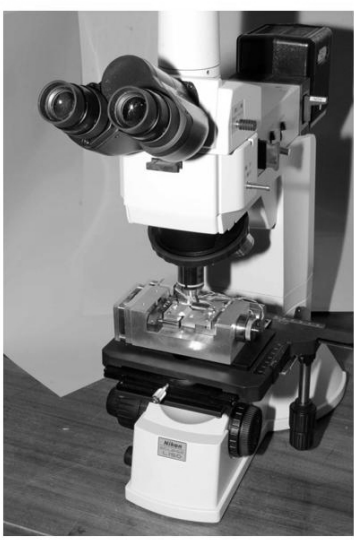

(b)

Figure 1. Experimental device used to measure local strain. (a) The microcompression test machine with typical cortical bone sample. (b) The global device composed of the micro-machine and an optical microscope

The load and displacement of the cross head of the compression device were also recorded during this test. The strain field was derived from in-plane displacements determined by the Digital Image Correlation method. Details are given in (Hoc et al., 
2000). Precision of the local strain measurements depends on the accuracy of the determination of the displacement and of the space between measured points. To determine this precision, a specific procedure similar to that reported by (Nicollela et al., 2001), was performed on two images of the same area without loading. Using this procedure with 50 pixels grid spacing, 0.05 pixels accuracy of the displacement measurement was obtained. In the present study, the space between measured points was fixed at $200 \pm 15$ pixels for the strain measured inside the osteon and 100 pixels for the strain map; this arrangement resulted in $5.10^{-4}$ and $1.10^{-3}$ strain accuracy, respectively.

\section{Experimental results}

\subsection{Heterogeneity of mineral content}

Figure 2 shows the grey level observations obtained with a SEM in backscattered electron mode (BSE) for two samples under longitudinal or transversal loading conditions. The linear relationship between the BSE grey levels and the mineral content is well established (Roschger et al., 1995; Bloebaum et al., 1997). Therefore tissues with low mineral content appear much darker than high mineral content ones.

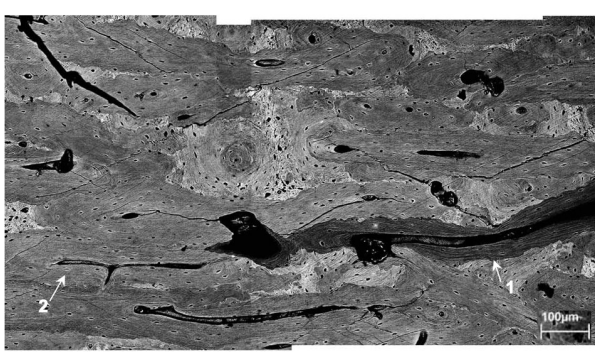

(a)

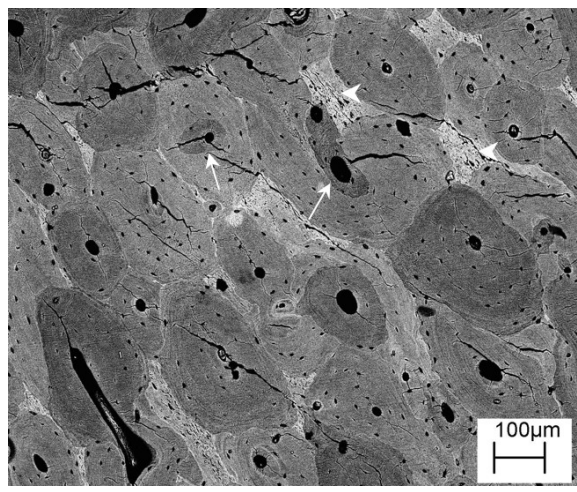

(b)

Figure 2. Image of the microstructure in a SEM-FEG in backscattered electron mode: (a) under longitudinal loading (b) under transversal loading

Figure 2 shows remodelling effect on mineral content heterogeneity among osteons. For instance, in Figure 2a a growing osteon with a low mineral content (labelled 1), compared to an older osteon (labelled 2) can be observed. The interstitial matrix (arrowheads Figure 2b), is composed of a mixture of old primary bone and fragments of remodelled osteons. This interstitial matrix is more 
mineralized than the osteons. "Resting lines" (arrows in Figure 2b) are also found in some osteons. These lines are the result of some delay in the remodelling process. The distribution of the mineral content is very heterogeneous between osteons but rather homogenous inside a single osteon. In the longitudinal direction, osteons are more or less straight and of about a millimeter length. Bone porosity, due to Haversian and Volkmann canals, is close to $5 \%$. In a simplified consideration, the microstructure of cortical bone is composed of densely packed concentric lamellar structures (osteons) embedded in an interstitial matrix.

\subsection{Local axial strain}

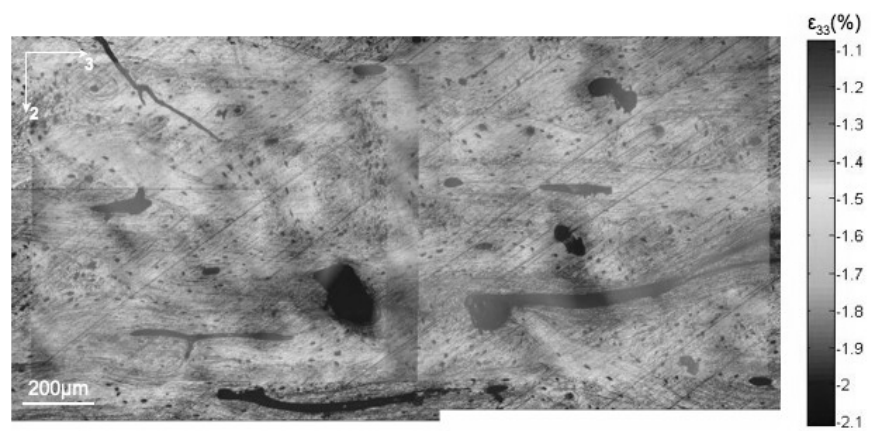

(a)

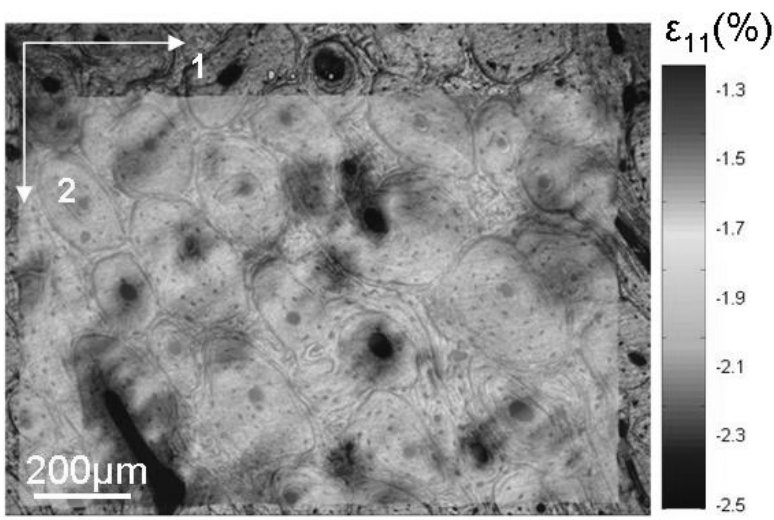

(b)

Figure 3. Map of local axial strain using digital image correlation and a regular mesh for measurements points. Axial strain is expressed in percent. (a) Longitudinal direction (b) transversal direction 
Figure 3 shows the maps of the local axial strains automatically provided by the microextensometry technique. In the present study, a regular mesh with a 50 pixels space grid was used to compute local strains $\varepsilon_{11}$ (Figure 3a) and $\varepsilon_{33}$ (Figure 3b). $\varepsilon_{11}$ corresponds to the local axial strains measured in the transversal direction, while $\varepsilon_{33}$ corresponds to the local axial strains measured in the longitudinal direction.

As noticed by several authors (Cowin, 2001; Currey, 2002), the macroscopic mechanical behaviours in the longitudinal and the transversal directions are quite different. The macroscopic strain, in Figure $3 \mathrm{a}$, measured in the longitudinal direction is $-1.34 \%$ with a corresponding macroscopic stress close to $150 \mathrm{MPa}$. In the transversal direction, in Figure $3 \mathrm{~b}$, the macroscopic strain is $-1.78 \%$ and the macroscopic stress is $95 \mathrm{MPa}$.

The two maps of axial strains show heterogeneity in the strain pattern. In the longitudinal direction, the minimum is close to $-1.1 \%$ and the maximum close to $-2.1 \%$. In the transversal one, the minimum is close to $-1.3 \%$ and the maximum close to $-2.4 \%$. In both cases, strain is mainly related to the Haversian microstructure, especially in the transversal direction where high contrast is observed. High local strains are also observed close to the Haversian canals and could be due to the presence of voids.

\subsection{Relationship between mineral content and local axial strain}

A correlation between the local axial strain and the grey levels obtained by backscattered electron imaging was examined. In order to avoid effect of voids, a specific procedure was used to compute local strains inside the microstructure. The local strain and the grey level were computed over sixty-three boxes in the transversal direction and fifty-height boxes in the longitudinal direction, distributed over the surface of the sample. Each box used in the computations covered a unique osteon but was free of Haversian or Volkmann canals and contained no crack. The distribution of the box length was $200 \pm 15$ pixels.

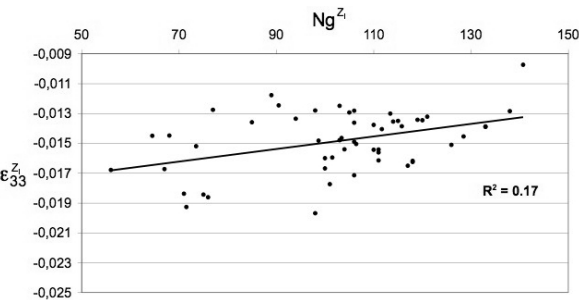

(a)

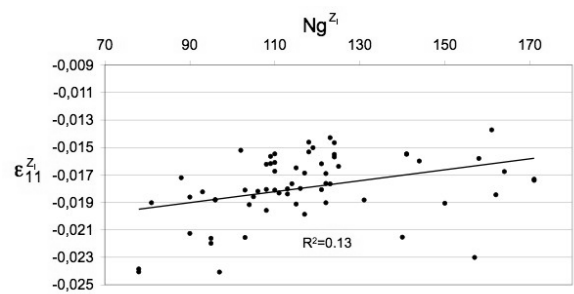

(b)

Figure 4. Relationship between normalized grey level and axial local strain. (a) Computed at $-1.34 \%$ of macroscopic strain in the longitudinal direction (b) Computed at $-1.78 \%$ of macroscopic strain in the transversal direction 
This relationship was achieved on Figure 4, by plotting the absolute values of the local axial strains computed in each box against their average normalized grey level measured using SEM. This figure showed that the absolute value of the strain increased when the grey level decreased in the two directions. However, linear regression analyses gave a low coefficient of correlation, $r^{2}=0.17(p<0.002)$ or $r^{2}=0.13(p<0.005)$. This implies that the mineral content is not predictive of the local strain.

\section{Mechanical behaviour modelling using microextensometry data}

\subsection{Identification procedure}

This procedure consists in a quantitative comparison between experimental and simulated data. In the present study, the experimental data were recorded during a tensile test in the transversal direction at $-1.78 \%$ macroscopic strain. The simulated data were obtained using finite element simulations performed on the actual cortical bone microstructure.

The mesh of the microstructure is given in Figure 5a. The shape of osteons was determined using the gradient of the grey level observed on a BSE image. The Haversian and Volkman canals were defined as free boundaries. Due to specific osteons geometry (see section 3.1), this two dimensional mesh was extruded in the 3direction, resulting in two layers of 13200 6-nodes linear triangular prismatic elements. In the present study, Abaqus V6.5 was used. In order to subject the actual cortical bone sample to the same boundary conditions as the experimental one, applied finite element boundary conditions were derived from experimental displacements measured using the DIC method (Hoc, et al, 2003). The primary bone areas (in grey on Figure 5a) were not meshed in the model but experimental displacements were applied on its frontiers.

a)

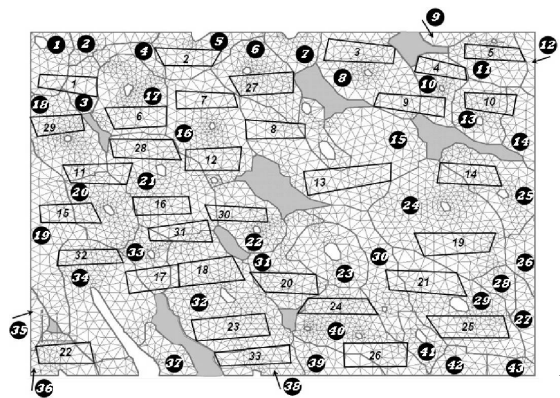

b)

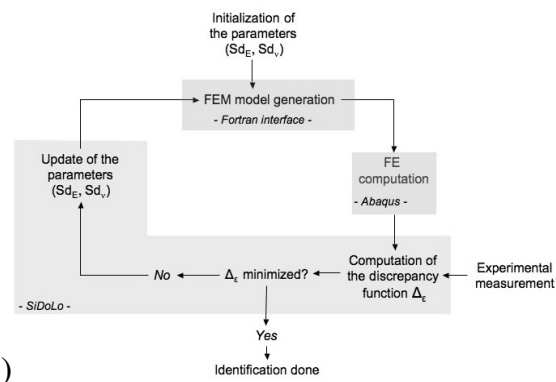

Figure 5. a) Mesh of the actual microstructure obtained using an SEM image. The 33 boxes correspond to areas where local strain was identified. The 43 circles correspond to osteon number. The white zones are Haversian or Volkman canals and grey zones are primary bone. b) Algorithm used for identification 
In the present study, based on nanoindentation works of Hoc et al. 2006, the material used in finite element simulations was assumed isotropic and linearly elastic in each element. Then, the Young's modulus $E^{i}$ and the Poisson ratio $v^{i}$ in each element (i) are given by:

$$
E^{i}=a G^{i}+b \text { and } v^{i}=c G^{i}+d
$$

where $G^{i}$ is the average grey level on element (i) and the quadruplet (a,b,c,d) are four constants. In order to have a better physical representation, we identified the four parameters: the mean Young's modulus $(\bar{E})$, the mean average Poisson ratio $(\bar{v})$ and their standard deviations $\left(\mathrm{Sd}_{\mathrm{E}} ; \mathrm{Sd}_{v}\right)$, which is an equivalent problem to the identification of $(a, b, c, d)$.

Experimentally, two sets of data are available. The first one is derived from the macroscopic analysis (stress-strain curve and macroscopic Poisson's ratio) and used to optimize the mean values $(\bar{E}, \bar{v})$. The second one consists of the local axial strain values computed in the thirty-three boxes used to optimize $\left(\mathrm{Sd}_{\mathrm{E}} ; \mathrm{Sd}_{v}\right)$. The identification procedure is described in Figure 5. In order to compute the discrepancy between simulated and measured local strain, the function $\Delta \varepsilon$, which corresponds to the total number of experimental errors, was introduced by:

$$
\Delta \varepsilon=\sum_{i} \max \left(\frac{\left|\varepsilon_{F E}^{i}-\varepsilon_{\mathrm{exp}}^{i}\right|}{0.0005}-1,0\right)
$$

Where $\varepsilon_{F E}^{i}$ and $\varepsilon_{\text {exp }}^{i}$ are respectively the computed and measured axial strain, in the box $\mathrm{i}, \max (\alpha, \beta)$ returns the highest value between $\alpha$ and $\beta$, and 0.0005 is the local strain measurement accuracy. In this formulation, a discrepancy lowers than experimental accuracy is not taken into account. Identification was performed through the automatic software SiDoLo (Pilvin, 1998).

\subsection{Numerical results}

Identification results are given in Figure 6. On this graph, level sets correspond to the discrepancy $\Delta \varepsilon$ between experimental and simulated strain field computed in the 33 boxes. Simulation performed with $\mathrm{Sd}_{\mathrm{E}}=\mathrm{Sd}_{v}=0$ shows a discrepancy close to 50 (point A). In this simulation, the material is considered elastic and homogenous in each element with a mean Young's modulus close to $5400 \mathrm{MPa}$ and a Poisson ratio close to 0.22 . Introducing heterogeneity by equation (1) does not affect the mean identified values but improved the discrepancy significantly to 34 when $\mathrm{Sd}_{\mathrm{E}}$ is close to $1100 \mathrm{MPa}$ and $\mathrm{Sd}_{v}$ close to 0.08 . In the present study, height sets of initial parameters $\left(\mathrm{Sd}_{\mathrm{E}}, \mathrm{Sd}_{v}\right)$ were used. The resulting relative difference between each optimized parameter of the height sets is less than $10 \%$. Similarly, a mesh refinement with 102200 elements does not significantly modify the simulation results. 


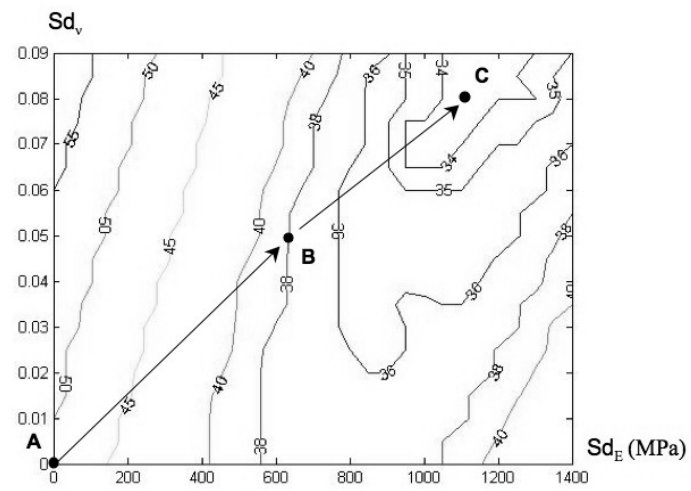

Figure 6. Discrepancy between experimental and simulated strain field according to Young's modulus standard deviation et $t_{E}$ and Poisson's ratio standard deviation et . Point A corresponds to the homogeneous solution and point $C$ corresponds to the best identified solution

If in the present study, the parameter $\mathrm{Sd}_{\mathrm{E}}$ is well defined, only a range of values for $\mathrm{Sd}_{v}$ can be extracted. This is principally due to the definition of the discrepancy function which takes into account only the local axial strain. The accuracy on the local transversal strain is too low to use it in the discrepancy function.

\section{Discussion and conclusion}

The results of this study present an inverse approach in order to determine the mechanical behaviour at the osteon scale using the measurement of the local strain field by microextensometry. The knowledge of the mechanical behaviour at this scale brings new insights into the local processes that are linked to the mechanical states such as damage, bone remodelling, or cell activity.

Experimental results obtained using DIC and SEM imaging show that cortical bone is a very heterogeneous tissue. The bone remodelling mechanism leads to a strong mineral content heterogeneity, which is more pronounced between osteons than inside the osteons themselves. Analysis of the mineral content in the longitudinal and transversal directions showed the specific geometry of osteons, which can be considered as straight hollowed tubes with an axis along the longitudinal direction. In a mechanical point of view, the local axial strain is also heterogeneous whatever the direction of loading is chosen. Analysis of the relationship between mineral content and the axial strain measured in the same area showed that the local absolute value of the strain increased when the content mineral decreased. However, a linear regression analysis in the two loading directions gave low correlation coefficients close to 0.15 . Consequently, the bone mineral content 
distribution is not the only parameter governing local strain; the effect of the microstructure must be accounted, using finite element simulations.

One major advantage of the microextensometry technique is to provide a map of the local strain field and consequently a lot of experimental local data, which are often beyond the scope of the macroscopic mechanical approach. On the other hand, the amazing increase of numerical computation capabilities has made possible the simulation of large actual microstructures and the derivation of local mechanical fields through the use of the finite element method. Consequently, significant comparisons can now be made between accurate strain field measurements and their simulated counterpart. In the present study, an inverse method and a very simple linear behaviour law were used to perform these comparisons. This law based on the work of Hoc et al (Hoc et al., 2006) takes into account the mineral content heterogeneity. However, the three types of osteons described by Ascenzi (Ascenzi et al., 1990) were excluded from this work. With this simplified approach, an improvement was obtained in the stress and strain concentration inside the microstructure. In particular, we show that, introducing the heterogeneity of mineral content enables to better reproduce the experimental strain field.

To summarize the present study, the microextensometry technique is very suitable for accessing mechanical heterogeneity at the osteon scale according to microstructure or disease parameters. For instance, it is well known that the microstructure of osteoporosic bone is completely different from the microstructure of healthy bone (Bousson et al., 2001). Finally to improve fracture risk prediction or analyse the effect of the remodelling process, the mechanical microstructural heterogeneity which is certainly one trigger parameter, should be introduced in future works.

\section{Acknowledgments}

The authors are grateful for the research support of the Ecole Centrale Paris. The authors are also grateful to the CNRS for a Post-doctoral fellowship. We are also grateful to Doctor Alain Meunier for his comments and suggestions.

\section{References}

Ascenzi A., Baschieri P., Bienvenuti A., "The bending properties of single osteons", $J$. Biomechanics, vol. 23, 1990, p. 763-771.

Bloebaum R.D., Skedros J.G., Vajda E.G., Bachus K.N., Constantz B.R., "Determining mineral content variations in bone using backscattered electron imaging", Bone, vol. 20, 1997, p. 485-490.

Bousson V., Meunier A., Bergot C., Vicaut E., Rocha M.A., Morais M.H., Laval-Jeantet A.M., Laredo J.D., "Distribution of intracortical porosity in human midfemoral cortex by age and gender”, J. Bone Miner. Res., vol. 16, 2001, p. 1308-1317. 
Burr D.B., Forwood M.R., Fyhrie D.P., Martin R.B., Schaffler M.B., Turner C.H., "Bone microdamage and skeletal fragility in osteoporotic and stress fracture", J. bone Miner. Res., vol. 12, 1997, p. 6-15.

Cowin S.C., Handbook of bone mechanics, Boca Raton, CRC Press, 2001.

Currey J.D., Bones: structure and mechanics, Princeton, Princeton University Press, 2002.

Currey J.D., "How well are bones designed to resist fracture", J. bone Miner. Res., vol. 18, 2003, p. 591-598.

Doumalin P., Bornert M., Crépin J., "Characterization of the strain distribution in heterogeneous materials", Mécanique et Industrie, vol. 4, 2003, p. 607-617.

Ho ba tho M.C, Rho J.Y., Ashman R.B., Atlas of mechanical properties of human cortical and cancellous bone, Van der perre G., Lowet G., Borgwardt A. (Eds), In vivo assessment of bone quality by vibration and wave propagation techniques Part II. ACCO Publishing, Leuwen, 1991, p. 7-32.

Hoc T., Rey C., Viaris de Lesegno, "Mesostructure of the localization in prestrained mild steel”, Scripta Materialia, vol. 42, 2000, p. 749-754.

Hoc T., Crepin J., Gelebart L., Zaoui A., "A procedure for identifying the plastic behavior of single crystals from the local response of polycrystals", Acta Materialia, vol. 51, 2003, p. $5477-5488$.

Hoc T., Henry L., Verdier M., Aubry D., Sedel L., Meunier A., "Effect of microstructure on the mechanical properties of Haversian cortical bone", Bone, vol. 38, 2006, p. 466-474.

Katz J.L., The structure and biomechanics of bone In mechanical properties of biological materials, edited by J.F. Currey and J.F.V. Vincent, Cambridge university press, Cambridge, 1980, p. 137-168.

Nicollela D., Nicholls A., Lankford J., Davy D., "Machine vision photogrammetry: a technique for measurement of microstructural strain in cortical bone", J. Biomechanics, vol. 34, 2001, p. 135-139.

Pilvin P., Notice d'utilisation de Sidolo version 2.4. Technical report, Décembre 1998, Ecole Centrale Paris.

Rho J., Zioupos P., Currey J.D., Pharr G.M., "Variations in the individual thick lamellar properties within osteons by nanoindentation", Bone, vol. 25, 1999, p. 295-300.

Roschger P., Plenk Jr. H., Klaushofer K., Eschberger J., "A new scanning electron microscopy approach to the quantification of bone mineral distribution: backscattered electron image grey-levels correlated to calcium Ka-line intensities", Scanning Microscopy, vol. 9, 1995, p. 75-88. 